\title{
Decay channels in the dissociative electron attachment to NO
}

\author{
Michael Allan \\ Department of Chemistry, University of Fribourg, chemin du Musée 9, 1700 Fribourg, \\ Switzerland
}

Received 10 September 2004

Published 26 October 2004

Online at stacks.iop.org/JPhysB/37/L359

doi:10.1088/0953-4075/37/21/L02

\begin{abstract}
Kinetic energy spectra of $\mathrm{O}^{-}$from dissociative electron attachment (DEA) to NO were measured at 7.6, 8.0, 9.0 and $10.0 \mathrm{eV}$ electron energy using a spectrometer with hemispherical energy selectors for both the incident electrons and the resulting ions. The measurements were performed at $10^{\circ}, 30^{\circ}, 90^{\circ}$ and $135^{\circ}$. The capacity of the spectrometer to detect both slow and fast ions was verified and the spectra were corrected for the analyser response function. Under these conditions only a signal corresponding to $\mathrm{O}^{-}$in the ground state and $\mathrm{N}$ in the first excited state was observed, a signal from the remaining two energetically accessible channels was absent. These observations confirm the original conclusion of Chantry (1968 Phys. Rev. 172 125) and reduce the range of possible explanations for the diametrically different observations of Orient and Chutjian (1995 Phys. Rev. Lett. 74 5017).
\end{abstract}

\section{Introduction}

Three channels are energetically accessible for dissociative electron attachment to NO around $E_{i}=10 \mathrm{eV}$,

$$
\begin{aligned}
& \mathrm{NO}\left({ }^{2} \Pi_{\Omega}\right)+\mathrm{e}^{-}\left(E_{i}\right) \longrightarrow \mathrm{O}^{-}\left({ }^{2} \mathrm{P}\right)+\mathrm{N}\left({ }^{4} \mathrm{~S}\right) \quad\left(E_{\mathrm{th}}=5.036 \mathrm{eV}\right) \\
& \longrightarrow \mathrm{O}^{-}\left({ }^{2} \mathrm{P}\right)+\mathrm{N}\left({ }^{2} \mathrm{D}\right) \quad\left(E_{\mathrm{th}}=7.420 \mathrm{eV}\right) \\
& \longrightarrow \mathrm{O}^{-}\left({ }^{2} \mathrm{P}\right)+\mathrm{N}\left({ }^{2} \mathrm{P}\right) \quad\left(E_{\mathrm{th}}=8.611 \mathrm{eV}\right),
\end{aligned}
$$

where the threshold energies $E_{\text {th }}$ are calculated from the dissociation energy of NO $\left(D_{0}=6.497 \mathrm{eV}[1]\right)$, the electron affinity of $\mathrm{O}(1.461 \mathrm{eV}[2])$ and spectroscopic data for $\mathrm{N}$ (2.384 eV and $3.576 \mathrm{eV}$ for the ${ }^{2} \mathrm{D}$ and ${ }^{2} \mathrm{P}$ states, respectively [3]). Both electronic fine structure states of the target, $\Omega=1 / 2$ and $3 / 2$, are thermally populated in the present experiment [4]. 
Rapp and Briglia [5] observed a band in the $\mathrm{O}^{-}$production in the $7-10 \mathrm{eV}$ electron energy range and measured the peak absolute value of the cross section to be $1.12 \times 10^{-18} \mathrm{~cm}^{2}$ (a 50\% higher value was obtained more recently [6]).

Chantry [7] obtained information on which of the channels are actually occurring by measuring the energies of the $\mathrm{O}^{-}$ions using a Wien filter and comparing the results to the values expected for each of the three channels (1-3) using the relation $E_{\mathrm{ion}}=(1-\beta)\left(E_{i}-E_{\mathrm{th}}\right)$, where $\beta$ is the ratio of the ion and molecular masses, $E_{i}$ is the incident electron energy and the values of the threshold energies $E_{\text {th }}$ are given in equations (1)-(3). He concluded that only channel (2) is operative.

More recently, Orient and Chutjian [8] reported the occurrence of all three channels, channel (1) being most abundant. The cause of the dramatically different results of the two experiments has not yet been resolved. The principal differences between the two studies lie in the experimental method of measuring $E_{\mathrm{ion}}$, and in the presence of a strong magnetic field, $6 \mathrm{~T}$, in the experiment of Orient and Chutjian. (Chantry used only a weak magnetic field, $\sim 10 \mathrm{mT}$, to collimate the electron beam.) Chantry used a Wien filter to measure the ion energies, whereas Orient and Chutjian used a novel device where ions drift on a trochoidal path in crossed axial magnetic and perpendicular electric fields. This device resembles the trochoidal monochromator (TM) $[9,10]$ often used for electrons, but a closer inspection reveals that its operating mode is entirely different. Standard TM relies on the use of only a weak magnetic field to reject charged particles with appreciable transverse momenta so that only particles with an essentially forward momentum pass. This by-passes an inherent weakness of the TM, namely that it filters the particles only with respect to forward momentum. In contrast, the very strong magnetic field used by Orient and Chutjian allows even particles with large transverse momenta to pass their device.

Orient and Chutjian emphasized in their paper [8] that their device, in contrast to that of Chantry [7], has the advantage of collecting ions of all kinetic energies without being dependent on the use of an extraction voltage. This implies the possibility that the extraction voltage used by Chantry could have been too low to collect fast ions and that the difference between the two findings could be due to an imperfection of Chantry's experiment.

This uncertain situation prompted two additional studies. Chu et al [11] measured the ion kinetic energies by means of ion time-of-flight and concluded, in agreement with Chantry, that channel (2) is dominant and channel (1) does not occur in their experiment. They report evidence for a weak occurrence of channel (3), however. They also argue against the occurrence of channel (1) on the basis of absence of ion signal below $7.42 \mathrm{eV}$, the threshold of channel (2). The latter argument has also been presented by Denifl et al [12], who measured the cross section with an instrument using hemispherical selectors for the electrons, that is, in a complete absence of any magnetic field. They used various extraction voltages and also concluded that channel (1) is absent in their experiment.

The discrepancy between the two results and the absence of a clear explanation has prompted a comment by Illenberger and Märk [13] and a reply by Orient and Chutjian [14]. Orient and Chutjian point out in their reply that differences in angles of observation could also be the cause of the difference of the two observations and that their device is superior in this respect because it collects ions ejected into all angles.

The issue thus remains unresolved and a clarification would be desirable for two reasons. The discrepancy casts doubt at the reliability of the various techniques to collect ions and measure their kinetic energies. If the results of Chantry were in error, it would cast doubt on the results obtained over the years for many molecules with commonly used instruments. On the other hand, if all experiments could be proven to be correct, it would reveal an interesting 


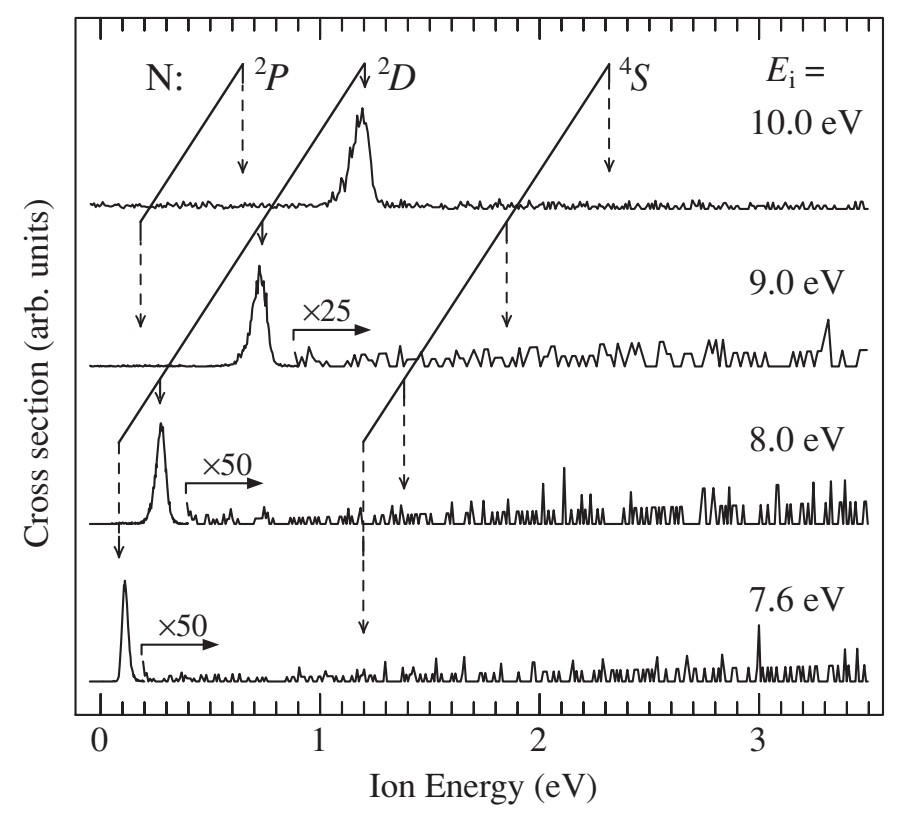

Figure 1. $\mathrm{O}^{-}$ion kinetic energy spectra collected at $90^{\circ}$. Energies at which ions from the processes (1), (2) and (3) are expected are indicated by arrows. Peaks are actually observed only at the energies corresponding to $\mathrm{N}$ atom in the ${ }^{2} \mathrm{D}$ state, that is channel (2). The spectra were corrected for the analyser response function but no background was subtracted. The background to the right of the $\mathrm{O}^{-}$peaks is shown vertically expanded to emphasize the absence of peaks due to channel (1).

dramatic sensitivity of the physics of the dissociative electron attachment to NO on a strong magnetic field.

The present study aims at making a positive test of the results of Chantry [7] and the subsequent studies $[11,13]$ by presenting ion kinetic energy measurements in the absence of magnetic field. It extends existing studies in an important way because observations are made at various angles, with superior signal-to-noise ratio, and at high resolution for the analysis of the ion kinetic energies. An additional important point is that the response of the present experiment as a function of ion energy has been calibrated on elastic scattering in helium and the spectra presented here are corrected for this response function. This excludes the problem of an inappropriate draw-out field, that is, the danger of missing ions of certain kinetic energies.

\section{Experiment}

The measurements were performed using a spectrometer with hemispherical analysers which has already been described $[15,16]$. The resolution in the incident electron beam was about $15 \mathrm{meV}$ and the beam current was about $1 \mathrm{nA}$. The response function of the spectrometer, that is the relative sensitivity of the instrument as a function of the kinetic energy of the detected particle, was determined on the elastic scattering in helium and the spectra shown in figure 1 are corrected for this response function. A small Wien filter separates ions and scattered electrons, in a way similar to that used by Hall et al [17]. The ion kinetic energy mode of the present instrument has already been tested [18]. 
Thermal broadening is an important aspect of the measurement of ion kinetic energies. The ion kinetic energy in the present experiment is substantially larger than $k T$ so that it may seem that thermal broadening is negligible. Chantry and Schulz $[19,20]$ have shown, however, that thermal broadening is 'amplified' in dissociative attachment, generally exceeds the resolution of the energy analyser ( $15 \mathrm{meV}$ in the present case) and dominates the measured distribution except at very low ion energies (see also [21]). For a stationary sample gas, Chantry and Schulz [20] obtained the equation

$$
W_{1 / 2}=\left(11 \beta k T E_{\text {ion }}\right)^{1 / 2}
$$

for the width $W_{1 / 2}$ of the ion peak. As an example, the width of the peak at $E_{i}=9.0 \mathrm{eV}$ in figure $1\left(E_{\text {ion }}=0.737 \mathrm{eV}\right)$ should be $W_{1 / 2}=0.33 \mathrm{eV}$, much more than $k T$ !

In the present experiment the thermal broadening is substantially reduced in comparison to a stationary sample, despite the fact that only an effusive nozzle was used. The reason is that the overlap of the narrow incident beam and the analyser acceptance cone (each about $0.25 \mathrm{~mm}$ in diameter) defines only a very small effective collision volume, within which the NO molecules move all in essentially the same direction. The width of the $\mathrm{O}^{-}$peak obtained with $E_{i}=9.0 \mathrm{eV}$ and shown in figure 1 is $75 \mathrm{meV}$, substantially less than the $330 \mathrm{meV}$ predicted for a stationary sample, but more than the $15 \mathrm{meV}$ resolution of the analyser. This indicates that some thermal broadening persists, primarily due to the finite size of the effective interaction volume and the nozzle exit, giving rise to a small but finite range of angles between the trajectories of the molecules and electrons. The high resolution in the ion kinetic energies is important because it avoids the problem of overlapping bands and helps distinguishing a narrow band from a broad underlying background.

\section{Results and discussion}

The spectra in figure 1 show clearly that signal is observed only for channel (2) and fully confirm the initial conclusion of Chantry [7]. There is a weak and approximately constant background to the right of the channel (2) $\mathrm{O}^{-}$peak, due presumably to channeltron dark count and stray electrons. This background does not have the shape of a narrow peak and thus cannot correspond to $\mathrm{O}^{-}$from channel (1). The spectra put an upper limit on the $\mathrm{O}^{-}$intensity from channel (1): it is at least $200 \times$ weaker than the $\mathrm{O}^{-}$intensity from channel (2) at 7.6 and $8.0 \mathrm{eV}$, at least $100 \times$ weaker at $9.0 \mathrm{eV}$ and at least $20 \times$ weaker at $10.0 \mathrm{eV}$. No narrow $\mathrm{O}^{-}$ peaks are observed also to the left of the $\mathrm{O}^{-}$peak- the present experiment gives no indication of signal from channel (3). The upper limit for this channel is about 1/20 of channel (2) signal at $10.0 \mathrm{eV}$.

Ions were collected at $90^{\circ}$ with respect to the electron beam in figure 1 . The measurements were repeated with the detector placed at $10^{\circ}, 30^{\circ}$ and $135^{\circ}$ and no signal due to channels (1) and (3) has been observed at any of these angles. The observed channel (2) $\mathrm{O}^{-}$signal was found to peak at $90^{\circ}$ and to decrease towards larger and smaller angles as already reported by Van Brunt and Kieffer [22]. The present experiment thus excludes, within the limits given above, the idea that channels (1) and (3) are active, at least in the absence of a strong magnetic field in the collision region. The hypothesis that these channels were missed in the earlier studies $[7,11,12]$ because of limited angular range of observation or because of ion kinetic energy discrimination due to problems with ion draw-out field is not justified.

The large selectivity with respect to the various channels remains puzzling, despite the substantial information existing on the Feshbach resonances in NO [23, 24]. Dissociative electron attachment to NO which has been laser excited to the $A^{2} \Sigma^{+}$Rydberg state results in a very high yield of $\mathrm{O}^{-}$and $\mathrm{N}$ in their ground states [25], that is, laser excitation activates 
channel (1). It seems that the formation of the resonance which mediates the laser activated process is strongly forbidden in electron attachment to ground state NO.

\section{References}

[1] Huber K P and Herzberg G 1979 Molecular Spectra and Molecular Structure IV. Constants of Diatomic Molecules (New York: Van Nostrand Reinhold) p 466

[2] Andersen T, Haugen H K and Hotop H 1999 J. Phys. Chem. Ref. Data 281511

[3] NIST Atomic Spectra Database http://physics.nist.gov/cgi-bin/AtData/main_asd

[4] Allan M 2004 Phys. Rev. Lett. 93063201

[5] Rapp D and Briglia D D 1965 J. Chem. Phys. 431480

[6] Krishnakumar E and Srivastava S K 1988 J. Phys. B: At. Mol. Opt. Phys. 21 L607

[7] Chantry P J 1968 Phys. Rev. 172125

[8] Orient O J and Chutjian A 1995 Phys. Rev. Lett. 745017

[9] Stamatović A and Schulz G J 1968 Rev. Sci. Instrum. 391752

[10] Allan M 1989 J. Electron Spectrosc. Relat. Phenom. 48219

[11] Chu Y, Senn G, Scheier P, Stamatović A, Märk T D, Brüning F, Matejcik S and Illenberger E 1998 Phys. Rev. A 57 R697

[12] Denifl G, Muigg D, Stamatović A and Märk T D 1998 Chem. Phys. Lett. 288105

[13] Illenberger E and Märk T D 1999 Phys. Rev. Lett. 824364

[14] Orient O J and Chutjian A 1999 Phys. Rev. Lett. 824365

[15] Allan M 1992 J. Phys. B: At. Mol. Opt. Phys. 251559

[16] Allan M 1995 J. Phys. B: At. Mol. Opt. Phys. 285163

[17] Hall R I, Čadež I, Schermann C and Tronc M 1977 Phys. Rev. A 15599

[18] Allan M, Asmis K R, Popović D B, Stepanović M, Mason N I and Davies J A 1996 J. Phys. B: At. Mol. Opt. Phys. 294727

[19] Chantry P J and Schulz G J 1964 Phys. Rev. Lett. 12449

[20] Chantry P J and Schulz G J 1967 Phys. Rev. 156134

[21] Dressler R A 1985 PhD Thesis Fribourg, unpublished

[22] Van Brunt R J and Kieffer L J 1974 Phys. Rev. 101633

[23] Gresteau F, Hall R I, Huetz A, Vichon D and Mazeau J 1979 J. Phys. B: At. Mol. Phys. 122925 Gresteau F, Hall R I, Huetz A, Vichon D and Mazeau J 1979 J. Phys. B: At. Mol. Phys. 122937

[24] Sanche L and Schulz G J 1972 Phys. Rev. A 669

[25] Kuo C T, Hardwick J L and Moseley J T 1994 J. Chem. Phys. 10111084 